\title{
ATIVIDADES INTERDISCIPLINARES E MULTIPROFISSIONAIS: RELATOS DE EXPERIÊNCIA COM PARTICIPANTES DO PROGRAMA DE PREVENÇÃO PARA DOENÇAS CARDIOVASCULARES
}

INTERDISCIPLINARY AND MULTIPROFESSIONAL ACTIVITIES CARDIOVASCULAR DISEASES PREVENTION PROGRAM EXPERIENCE REPORT

\section{Albertina Bonetti \\ Doutora em Enfermagem - UFSC \\ Professor titular - UFSC \\ tina@cds.ufsc.br}

Geny Aparecida Cantos Pós doutorado em Parasitologia Molecular pelo Centro de Investigações Biológicas de Madrid Professora Associada - UFSC geny@ccs.ufsc.br

Joselma Tavares Frutuoso Doutora em Engenharia de Produção - UFSC Professor adjunto- III - UFSC frutuoso@cfh.ufsc.br

Maria Edinéia Rocha Professora de educação física edineianatura@yahoo.com.br

\section{RESUMO}

O artigo apresenta a experiência realizada por uma equipe de saúde interdisciplinar e multiprofissional, tendo como foco um programa de prevenção para doenças cardiovasculares. No período de 2007 a 2009, participaram desta experiência 480 pessoas, portadoras ou não de doenças crônica, sendo que a maioria pertencia à comunidade universitária. Inicialmente, as pessoas tiveram um atendimento com nutricionista e cardiologista. Paralelamente, foram realizadas palestras mensais para o grupo sobre educação em saúde com temas relacionados ao processo de saúde e adoecimento. Ao final, visando unir medicina convencional a outros modelos assistenciais de promoção e prevenção à saúde, a equipe disponibilizou várias práticas corporais para serem somadas no enfrentamento da doença pelos pacientes. como: biodanza; práticas aquáticas (watsu, halliwick e biodanza) e vivências lúdicas do coração. Os participantes também tiveram atendimento psicológico em grupo, educação nutricional com almoços comunitários e visitas ao supermercado. O monitoramento dessas ações envolveu sobretudo questionários estruturados, relatos verbais, exames clínicos e bioquímicos. A análise dos dados foi organizada em categorias temáticas envolvendo aspectos que influenciavam na adesão ao tratamento assistencial. Os participantes relataram a importância de tomarem consciência de como estavam agindo, sendo possível planejar mudanças positivas em suas atitudes cotidianas, o que contribuiu para uma melhor qualidade de vida e bem-estar psicológico dos participantes do programa. Estas formas diferenciadas de tratamentos disponíveis nos diferentes grupos

\section{cc) (†) Esta obra está licenciada sob uma Licença Creative Commons.}


Atividades Interdisciplinares e Multiprofissionais: Relatos de Experiência com Participantes do Programa de Prevenção para Doenças Cardiovasculares

terapêuticos fortaleceram os valores humanistas associados à recuperação física e emocional dessas pessoas, integrando-os melhor com o mundo, com o outro e consigo mesmo. A experiência contribuiu para a construção do conhecimento e o crescimento profissional, valorizando as ações da equipe de saúde e oferecendo aos participantes um modelo assistencial com foco na promoção à saúde e prevenção de doenças.

Palavras-chave: Doenças cardiovasculares; Doenças crônicas; Promoção à saúde; Prevenção a doenças, Tratamentos alternativos; Modelos assistenciais de saúde.

\section{INTRODUÇÃO}

As doenças crônicas não-transmissíveis (DCNT) são importantes causadoras de mortes, com maior incidência nos grandes centros urbanos e em paises industrializados, embora o número de portadores esteja crescendo muito no mundo todo (LEITE et al.., 2002). São exemplos das mesmas: diabetes mellitus não insulino-dependente, hipertensão arterial, doenças cardiovasculares, osteoporose e alguns tipos de câncer, como o de cólon e o de mama (MOLINA et al., 2003). As doenças cardiovasculares têm se tornado a principal causa de morte no mundo, sendo responsáveis por $28 \%$ de todas as mortes (SILVA et al., 2010). Diversos fatores estão relacionados à mortalidade desta parcela da população: a magnitude da doença, a necessidade intensa de atuação dos cuidadores e a carência de projetos terapêuticos que permitam o acesso do portador aos serviços e ações integradas em todos os níveis de atenção à saúde (MALTA, 2010).

Os fatores de risco que contribuem para o aparecimento das doenças crônicas podem ser intrínsecos ao indivíduo, como histórico familiar e idade; ou extrínsecos, como estilo de vida sedentário, consumo excessivo de cigarros, álcool, café e uso de corticóides (SIMÃO, 2004). Essas doenças têm, em comum, uma evolução progressiva, causando, muitas vezes, limitações para realizar tarefas simples, sendo necessário somar ao tratamento medicamentoso formas alternativas de terapia para o tratamento global do organismo adoecido. Geralmente o paciente está submetido à terapêutica farmacológica recomendada que lhe é prescrita. Quando não ocorre adesão às intervenções clínicas farmacológicas, há uma premissa de que a causa deve-se à falta 
Atividades Interdisciplinares e Multiprofissionais: Relatos de Experiência com Participantes do Programa de Prevenção para Doenças Cardiovasculares

de conhecimento do paciente sobre a sua doença e sobre os cuidados de sua saúde integral. A consciência do processo saúde-adoecimento é afetada por aspectos relacionados à subjetividade e autonomia da pessoa ou cuidador bem como o meio social e cultural em que a pessoa está inserida (CIPOLLE, et al.. 2006; SERRANO et al.., 2009).

Muitos fatores influenciam a maneira de sentir-se ou não doente, em especial, as características psicológicas e culturais, há necessidade de mudança de paradigma que abra espaço para uma cultura médica mais abrangente e que atenda às necessidades do ser humano. Hoje, há evidências que apontam para a necessidade de mudança da cultura médica que, em nosso meio, tornou-se excessivamente medicamentosa e intervencionista. A medicina-convencional tem auxiliado no alívio de sintomas e no tratamento de muitas doenças. Contudo, em especial no tratamento de doenças crônicas e degenerativas, necessita contemplar a junção de modelos múltiplos de atenção à saúde que integre e harmonize o organismo (GOSTAWAMI, 2004).

Há uma distância grande entre o discurso preventivo sobre os riscos cardiovasculares e os resultados alcançados pela prática medicamentosa na vida de indivíduos que buscam mudanças positivas no seu estilo de vida e uma vida mais saudável. É um desafio realizar a integração de ações preventivas a essas doenças, pois são necessárias práticas que levem o indivíduo a conhecer e entender seu estilo de vida, suas necessidades, suas emoções, seus sentimentos, seus valores, suas expectativas e suas prioridades de vida, (VALLS; SILVA, 2008).

Assim, neste trabalho, serão relatadas experiências realizadas nesse projeto de extensão na área de prevenção para doenças cardiovasculares, envolvendo uma equipe interdisciplinar e multiprofissional. As ações iniciaram em 1997 com dois cardiologistas, dois nutricionistas e dois farmacêuticos bioquímicos. Nessa época, percebeu-se que havia elevado número de pacientes que passavam pelo setor de cardiologia do Serviço de Atendimento à Comunidade Universitária do Hospital Universitário da Universidade Federal de Santa Catarina (SASC-HU-UFSC). A cardiologia e a clínica geral recebiam em torno de 700 pacientes por mês. Assim, nesse mesmo ano, esta equipe começou a prestar atendimento cardiológico e nutricional aos indivíduos da comunidade universitária, tentando conscientizá-los via conversas a mudarem seus hábitos e estilo de vida não saudáveis, a fim de melhorar a qualidade de vida dos mesmos. Com o tempo, surgiu a necessidade de avaliar e acompanhar estes pacientes, foi criado então o projeto 
Atividades Interdisciplinares e Multiprofissionais: Relatos de Experiência com Participantes do Programa de Prevenção para Doenças Cardiovasculares

"Avaliação da intervenção multiprofissional e interdisciplinar na prevenção e tratamento de eventos cardiovasculares em uma comunidade universitária".

Nos primeiros cinco anos do projeto, os pacientes tinham acesso à educação nutricional (consultas com nutricionista); acompanhamento cardiológico (consultas e exames) no Hospital Universitário e palestras mensais sobre temas relacionados ao processo de saúde-adoecimento. Em função de constantes avaliações dos profissionais e as demandas dos participantes, outras modalidades de atendimento foram surgindo para melhorar e ampliar a qualidade de vida dos participantes do projeto.

Assim, em 2002, teve inicio a biodanza, que significa o movimento do corpo com ritmo da música e da vida (TORO, 2002). O objetivo foi produzir modificações estáveis no estilo de vida, diminuindo o estresse e elevando a qualidade de vida de pessoas com dislipidemia, por meio da música, do movimento e da comunicação em grupo. Esse projeto objetivou explorar também os limites e possibilidades da linguagem corporal, em suas múltiplas expressões e experiências nas relações consigo mesmo e com o outro, contribuindo para a capacidade de tocar, de sorrir, de abrir espaço para a troca de interações coletivas (CANTOS et al., 2005; CANTOS et al.., 2008).

Em 2004, surgiu o programa vivências lúdicas do coração com objetivo geral de proporcionar vivências corporais lúdicas para pessoas que apresentam diagnóstico de dislipidemia Outras metas eram estimular o desenvolvimento da criatividade; proporcionar prazer, autonomia, fantasia; possibilitar uma melhor compreensão e percepção qualificada do movimento; oferecer oportunidades às pessoas para, ao (re) descobrirem o seu corpo, (re) descobrir o seu significado e a sua importância de ser (BONETTI, 2006).

Em 2005, foram oferecidas atividades de watsu e halliwick para pacientes com doenças crônicas com objetivo era melhorar a qualidade de vida de pessoas tanto no aspecto físico como emocional. Em conjunto, são recomendadas para todos os tipos de pessoas em qualquer idade (crianças, adolescentes, jovens, adultos e idosos) e nos casos de estresse físico e mental, medos, osteoporose, problemas neurológicos, ortopédicos, bloqueios emocionais, DCV, etc. (BACKER et al.., 2000). O método watsu foi escolhido por ajudar as pessoas a "silenciarem a mente", 
liberando e flutuando o corpo na água de forma a harmonizá-lo, "dissolver" as tensões e fadigas, reduzir o estresse e a ansiedade, tornando inclusive o sono mais tranqüilo. $\mathrm{O}$ halliwick visava possibilitar o desenvolvimento de habilidades como respirar e controlar o corpo na água com segurança e alegria (SCHUTZ, 2009).

Em 2008, watsu e halliwick foram associados a biodanza aquática, a fim de utilizar outra linguagem corporal, onde a fala é silenciosa, priorizando-se o olhar, o toque, a carícia, o afeto e a valorização de si mesmo e também a valorização do outro, trazendo a percepção de totalidade (SCHUTZ, 2009)

$\mathrm{O}$ atendimento psicológico grupal teve inicio também em 2008 tendo como foco desenvolver vínculos afetivos e proporcionar um clima favorável a novas aprendizagens para a ampliação do repertório comportamental, favorecendo a auto-observação e o autoconhecimento (FRUTUOSO; CANTOS, 2009).

Nesse artigo, relatamos o percurso dessa experiência interdisciplinar e multiprofissional de treze anos que se apoiou em estratégias de promoção à saúde e prevenção de doenças cardiovasculares, envolvendo o tripé da integração: paciente, tratamento e equipe de saúde.

\section{METODOLOGIA}

\subsection{Participantes}

Entre os anos 1997 e 2009, participaram deste programa quatrocentos e oitenta pacientes, $80 \%$ deles funcionários, alunos ou professores da Universidade Federal de Santa Catarina (UFSC), os noventa e dois eram da comunidade local que tiveram acesso às diversas modalidades de tratamentos. Primeiramente, foram incluídos no projeto de extensão somente pessoas que apresentavam um quadro de dislipidemia ou doença cardiovascular. Posteriormente, em 2005, abriu-se espaço para cento e sessenta e oito participantes assintomáticos e aparentemente 
saudáveis, mas que apresentavam pelo menos um fator de risco coronário e ainda para mais trinta e cinco pessoas portadoras de outras doenças crônicas não-transmissíveis, tais como hipertensão arterial sistêmica, diabetes mellitus, fibromialgia, artrose e labirintite. A faixa etária do grupo variou de vinte a oitenta e nove anos, sendo que $81 \%$ tinham idade de entre cinquenta a sessenta e cinco anos e $79 \%$ eram do sexo feminino.

Participaram de cada grupo em torno de vinte e cinco pacientes, exceto o de atendimento psicológico que recebeu no máximo dez pessoas por dois semestres letivos. A terapia aquática teve caráter contínuo. A biodanza e as vivências lúdicas do coração foram semestrais, ou seja, a cada início de semestre foram criados grupos, com membros novos e também participantes do semestre anterior. Esta estratégia foi aplicada também ao grupo de atendimento psicológico a partir de 2009. Cada pessoa podia participar de uma ou mais atividades oferecidas pelo projeto de extensão.

Todas as terapias envolveram mediações bioquímicas (ROSEIN et al., 2004), incluindo os indivíduos assintomáticos. $\mathrm{O}$ agendamento e a coleta dos exames foram realizados por alunos de extensão e pesquisa da UFSC. No momento da coleta cada participante respondia o questionário de estresse proposto por LIPP et al. (1996).

Todos os participantes foram esclarecidos sobre o projeto e acerca dos programas de tratamentos alternativos oferecidos. Os participantes assinaram o termo de consentimento livre e esclarecido, conforme prevê a resolução 196/1996 que rege pesquisas realizadas com seres humanos (CONEP, 2000). Este trabalho teve a aprovação pela Comissão de Ética de Pesquisa em Humanos da Universidade Federal de Santa Catarina, registro no Mistério da Saúde: CONEP n.299/08.

\subsection{Níveis de ação}

As ações globais foram desenvolvidas em dois níveis: individualizado e em grupo. 
Atividades Interdisciplinares e Multiprofissionais: Relatos de Experiência com Participantes do Programa de Prevenção para Doenças Cardiovasculares

\section{a) Atendimento individualizado}

Primeiramente os pacientes passavam pelo exame clínico realizado pelo setor de cardiologia e após um "check-up" personalizado, mesmo que as pessoas não tivessem doenças, exames bioquímicos eram solicitados. Os exames mais complexos foram solicitados apenas quando os testes iniciais deixaram dúvida ou levantaram suspeitas de outras doenças.

Como importante estratégia de intervenção de doenças cardiovasculares, foi utilizada a educação nutricional desenvolvida por uma nutricionista do HU-UFSC e duas professoras do Departamento de Nutrição da mesma universidade. A avaliação do estado nutricional deu ênfase aos indicadores antropométricos, distribuição da gordura corporal e percepções, observadas por meio de perguntas formuladas a cada pessoa. A partir dessa avaliação, programou-se uma dieta adequada que considerava as especificidades de cada participante, pois o grupo era bastante heterogêneo (SILVEIRA et al..2009).

Tanto no setor de cardiologia como no de nutrição, as pessoas eram orientadas a adotar hábitos que pudessem atenuar os riscos de doenças crônicas e/ou a adotar comportamentos pró-saúde.

\section{b) Educação e saúde para ao grupo}

Desde o início do projeto, as pessoas foram convidadas a participar de palestras mensais, que tiveram duração de aproximadamente duas horas. Essas foram coordenadas pelos profissionais integrantes do projeto e outros profissionais convidados, seguindo escala pré-determinada, com assuntos referentes às doenças cardiovasculares e aos diversos fatores implicados no processo saúde-adoecimento.

Em 2008, dentro do programa da educação nutricional foi criada uma importante estratégia de intervenção de doenças cardiovasculares chamada de almoços "comunitários", que visou orientar e avaliar uma alimentação balanceada. Esta estratégia, por sua vez, foi realizada coletivamente uma vez por mês na residência de um dos participantes, com o objetivo de proporcionar melhor integração do grupo e promover hábitos alimentares saudáveis e trocas de experiências. O 
Atividades Interdisciplinares e Multiprofissionais: Relatos de Experiência com Participantes do Programa de Prevenção para Doenças Cardiovasculares

aprimoramento desta etapa realizou-se por meio de visitas ao supermercado com os pacientes, sob a supervisão da nutricionista na qual se explorou a viabilização e a adequação de uma dieta balanceada individualmente.

A equipe interdisciplinar e multiprofissional realizaram também sistematicamente reuniões semanais com o intuito de integrar a medicina convencional com outros modelos assistenciais e/ou alternativos, a fim de trabalhar com prevenção de doenças e promoção de saúde. As ações deliberadas por esta equipe tiveram como objetivo manter o organismo saudável, diminuindo a resposta do estresse negativo, um dos causadores de doenças.

Considerando o acima exposto, a equipe de profissionais passou a utilizar várias modalidades de intervenção, por meio de terapias alternativas, focando na deteç̧ão de sintomas emocionais e no bem-estar psicológico dos participantes.

\subsection{Práticas corporais e benefícios esperados}

Em 2002, foi criado o projeto "Efeito terapêutico da biodanza na restauração do equilíbrio e saúde mental de pacientes com dislipidemia" que inicialmente atendia vinte pessoas anualmente com dislipidemia. Em 2006, a fim de atender a comunidade local, um outro grupo foi criado. Cada um deles teve anualmente quarenta e sete encontros. Neste tipo de atendimento, as sessões foram realizadas uma vez por semana, com duração de duas horas, conduzidas por facilitadores de biodanza (CANTOS et al.., 2008).

Em 2004, o programa vivências lúdicas do coração foi desenvolvido por uma professora do Departamento de Educação Física da UFSC. Este tipo de atividade explorou o lúdico, a cooperação, a brincadeira e o prazer para realização de atividades físicas, as quais consistiram em danças, caminhadas, jogos, alongamentos, exercícios localizados, relaxamentos, vivências aeróbicas, etc. (BONETTI, 2006). Neste programa, as vivências foram realizadas três vezes por semana, com duração de uma hora e atendeu aproximadamente vinte e cinco pessoas por semestre. 
Atividades Interdisciplinares e Multiprofissionais: Relatos de Experiência com Participantes do Programa de Prevenção para Doenças Cardiovasculares

Em 2005, a hidroterapia, com os métodos watsu e halliwick, foi oferecida por profissional formada em Educação Física com especialização nas referidas técnicas. Em 2008, as técnicas de watsu e halliwick foram associadas com a biodanza aquática e ministradas pela mesma profissional que também era facilitadora de biodanza. Esta nova modalidade de atendimento fez parte de um subprojeto intitulado Vida com saúde: "o método watsu e halliwick biodanza aquática como agentes modificadores do diestresse (estresse negativo) de indivíduos com doenças crônicas". O trabalho foi conduzido de forma que as atividades vivenciadas pudessem fortalecer os valores humanistas associados à recuperação física e emocional desses pacientes, considerando que essas dimensões devem ser interligadas. Participaram deste trabalho trinta pacientes com doenças crônicas, os quais foram conduzidos a uma terapia em piscina aquecida durante duas horas, uma vez por semana. O programa teve a duração de doze meses (janeiro a dezembro de 2006), totalizando quarenta e oito sessões anuais (ROCHA et al.; 2008; SCHUTZ et al., 2009).

\subsection{Outra modalidade de atendimento}

Em 2007, o atendimento psicológico grupal passou a ser oferecido por professora do Departamento de Psicologia, aos pacientes encaminhados pelos serviços de cardiologia e nutrição. Porém a participação era voluntária. A pessoa tomava a decisão se queria ou não entrar no grupo de apoio psicológico. O modelo de terapia seguiu os princípios da Análise do Comportamento e da filosofia do Behaviorismo Radical de Skinner (MARTIN; PEAT, 2009; CATANIA, 1999). O foco desde tipo de atendimento está baseado na descrição e análise das contingências de reforçamento passadas que fizeram parte da história de vida de cada um ou das contingências vigentes que são responsáveis pelo modo de ser e agir das pessoas. O termo contingência envolveu no mínimo a análise e compreensão de três tipos de eventos: (1) eventos antecedentes (contexto, situações, condições), (2) eventos comportamentais (as ações, tudo que a pessoa faz, diz, sente e pensa) e (3) eventos conseqüentes (as conseqüências dos comportamentos que influenciam a própria pessoa e todo o seu ambiente: afetivo, social, familiar etc.). Em outras palavras, implica analisar simultaneamente as inúmeras possibilidades de relações funcionais existentes entre estes três eventos responsáveis pelo atual repertório comportamental. O grupo de apoio psicológico foi realizado ao longo de dois semestres letivos, ocorrendo trinta e quatro 
Atividades Interdisciplinares e Multiprofissionais: Relatos de Experiência com Participantes do Programa de Prevenção para Doenças Cardiovasculares

encontros, os quais eram semanais com duração de duas horas. O grupo era aberto à entrada de participantes até o terceiro encontro e depois fechado, com no máximo dez participantes.

\section{RESULTADOS E DISCUSSÃO}

Pessoas com doenças crônicas vivem seus problemas e desafios com períodos de melhora ou piora, nem sempre previsíveis, com ameaças e limitações em suas rotinas diárias, no trabalho, nos relacionamentos, etc. A prevenção cardiovascular tem sido baseada no conceito de redução de risco cardiovascular global, que envolve os esforços para prevenir ou reduzir novos eventos cardiovasculares (CANTOS, 2008). Diversos estudos demonstraram que é possível reduzir a carga de doença nesta população por meio de estratégias preventivas (GOSTAWAMI, 2004; BONETTI, 200; SILVEIRA et al., 2009).

Nesse trabalho, as consultas com cardiologistas eram a "porta de entrada" do paciente, os quais fizeram o diagnóstico clínico, elaboraram o plano terapêutico e realizaram os encaminhamentos necessários. Houve um esforço dirigido para as medidas preventivas de controle dos fatores de risco para doença arterial coronariana.

A realização de exames bioquímicos periódicos foi uma ferramenta importante para monitorar e avaliar as condições de saúde dos participantes desse projeto. O resultado das análises bioquímicas ofereceu informações diagnósticas que orientaram os procedimentos terapêuticos realizados (ROSEIN, et al.., 2004; CANTOS, et al.., 2008).

O tratamento das doenças crônicas, na verdade, requer o envolvimento dos cuidadores com estratégias preventivas e educacionais para que os resultados esperados sejam alcançados. Estamos acostumados a um modelo onde o terapeuta é o locutor e o paciente (conforme o próprio nome indica) é o receptor do conteúdo. Ferreira e colaboradores (2006) afirmam que o comportamento de adesão implica em o paciente identificar os resultados que pretende alcançar e programar contingências para que isto ocorra, tornando-se protagonista de seu tratamento. Por 
Atividades Interdisciplinares e Multiprofissionais: Relatos de Experiência com Participantes do Programa de Prevenção para Doenças Cardiovasculares

exemplo, em todas as atividades desenvolvidas pela equipe foi de fundamental importância a utilização de uma linguagem acessível ao paciente considerando as questões subjetivas de cada pessoa envolvida.

Um dos grandes desafios enfrentados pela equipe foi interferir positivamente no comportamento alimentar das pessoas para que as mesmas pudessem efetivar as mudanças necessárias na alimentação cotidiana. Isso acarretou grandes alterações no modo de pensar e agir desses pacientes, modificando, inclusive o componente emocional segundo relato dos pacientes. A avaliação nutricional realizada com os participantes desse programa representou uma tarefa importante para a equipe multidisciplinar, pois possibilitou a identificação de grupos de riscos e intervenções não apenas relacionadas com os efeitos, mas também com as causas da alimentação inadequada. Nossa experiência mostrou que apesar das pessoas estarem atentas aos cuidados indispensáveis ao bom estado nutricional, ainda é necessário que haja ações de caráter preventivo de doenças crônico-degenerativas associadas ao excesso de peso.

Nesse estudo, apesar dos esforços empreendidos, a adesão ao tratamento, não foi superior a $20 \%$. Houveram resistências que ainda se fazem presentes para os encaminhamentos. Talvez a raiz do problema esteja relacionada a variáveis relacionadas com situação de insatisfação pessoal de difícil mensuração, o que exige mudanças enormes e complexas em vários setores da vida da pessoa: profissional, afetivo, social, etc. No grupo estudado, por exemplo, ser portador de doença crônica configura-se um motivo de insatisfação, pois o indivíduo precisa se adaptar à atual condição física e conciliar isto ao seu desejo de ser aceito pela sociedade. Diante desde quadro, é preciso levar em consideração as limitações de cada um, para ser possível programar intervenções nutricionais mais adequadas.

Nesta direção, os almoços comunitários mensalmente realizados foram importantes estratégias empregadas. Além da parte de integração social, houve a orientação e a avaliação do cardápio trazido pelos participantes. Nesses encontros, percebeu-se que as refeições eram perfeitamente balanceadas, somente o açúcar utilizado em excesso nas sobremesas apresentava problema. Podese dizer que os almoços comunitários e as palestras educativas sem dúvida influenciaram positivamente na adesão da orientação alimentar (PASSOS; CANTOS, 2009). 
Atividades Interdisciplinares e Multiprofissionais: Relatos de Experiência com Participantes do Programa de Prevenção para Doenças Cardiovasculares

As reuniões semanais sistemáticas com os profissionais da equipe interdisciplinar foram também de grande valia para integrar as diversas formas de pensar e agir da equipe. Nelas, houveram discussões no sentido de rever conceitos, posturas, atitudes, condutas, provendo inovações na prática, trabalhando conflitos emergentes, de forma a facilitar os relacionamentos interpessoais entre os membros da própria equipe e da equipe com os pacientes.

Por conseguinte, as experiências que foram retratadas nesse trabalho deram ênfase na relação entre as condições de estresse e os diferentes tratamentos alternativos oferecidos para este tipo de população (ROSEIN, et al., 2004). Esta intervenção foi respaldada num conjunto de atividades: consultas cardiológicas, nutricionais, palestras educativas, almoços comunitários e grupos de apoio (atendimento psicológico, vivências do coração, biodanza e três tipos de práticas aquáticas).

Dentro desde contexto de cuidado e atenção à saúde, a equipe interdisciplinar e multiprofissional têm utilizado elementos quantitativos (exames bioquímicos) e componentes "subjetivos" (depoimentos e questionários de estresse) para monitorar o estado de saúde dos indivíduos que integraram o programa. Trabalhos realizados mostraram que as emoções negativas podem estar relacionadas com o aumento do colesterol total, fração LDL-colesterol, triglicerídeos e diminuição da fração HDL-colesterol, e que as atividades relacionadas com a promoção à saúde melhoram esses parâmetros (ROSEIN et al.., 2004; BONETT, 2006; CANTOS et al., 2008; SCHUTZ et al.. 2009).

$\mathrm{Na}$ esfera psicológica, a equipe interdisciplinar e multiprofissional percebeu que os padrões comportamentais e a história de vida de cada paciente são fatores que contribuem para o sofrimento e a resistência a mudanças nos hábitos alimentares e a adoção de estilos de vida mais saudáveis (ROSEIN et al., 2004 LIPP et al., 1996). A partir destas constatações, a equipe procurou contornar este problema oferecendo a seus participantes os tratamentos alternativos relatados nesse artigo. Entretanto, verificou-se que seis pessoas que apresentavam dificuldades interpessoais, padrão agressivo de interação e problemas afetivos que necessitavam de atendimento psicológico foram convidadas a participar, mas não aceitaram. Nos encontros, os atendimentos psicológicos ocorriam de forma gradativa, os membros espontaneamente relatavam 
Atividades Interdisciplinares e Multiprofissionais: Relatos de Experiência com Participantes do Programa de Prevenção para Doenças Cardiovasculares

seus problemas e dificuldades, cada participante era protagonista de suas escolhas e decisões e se beneficiava da interação com as outras pessoas, encontrando não só apoio emocional, mas também idéias e orientações para um novo estilo de vida (DEL PRETTE, DEL PRETTE, 2009). Ao longo desses anos, percebeu-se que a biodanza por meio da música, conto e expressão corporal nos encontros, repercutiu de forma integradora e afetiva nas interações dos membros que compõem o grupo. Os participantes relataram melhora dos sintomas e os exames apontam melhora também no quadro clínico e laboratorial dos mesmos (CANTOS et al.., 2008). Por exemplo, entre os anos 2002 a 2007, em torno de cento e cinquenta pessoas participaram do grupo de biodanza durante pelo menos dois anos. Dessas, vinte pessoas compareceram com freqüência acima de $80 \%$, indicando um bom índice de adesão em longo prazo. Percebeu-se que esse grupo de biondaza quando comparado ao grupo controle (pessoas que participavam do projeto, mas não faziam biodanza), apresentou melhoras no colesterol e frações bem como na glicemia e magnésio. Esses parâmetros bioquímicos tiveram boa correlação com estado de estresse (CANTOS, et al., 2005). Por outro lado, por meio das respostas ao questionário de estresse e de depoimentos, percebeu-se que a biodanza conduziu a melhoras no estilo e na qualidade de vida de seus integrantes, sobretudo na comunicação, na auto-estima e também na aceitação do outro com todas as suas diferenças (CANTOS et al.., 2008).

No desenvolvimento das vivências corporais lúdicas, percebeu-se grande receptividade por parte dos participantes em função da diversidade de atividades que este tratamento ofereceu como danças, ginásticas, caminhadas, relaxamentos, jogos recreativos, capoeira, ioga, entre outras. Observou-se no relato verbal também maior dinamismo nos movimentos, mais motivação das pessoas para se exercitar, ampliação quanto à execução de movimentos e bem-estar dos participantes. Não obstante, verificou-se que a motivação era determinante para que o indivíduo realizasse exercícios físicos regularmente, o que repercutiu na melhor execução das tarefas da vida diária e também na socialização entre os participantes. Além disso, foi possível perceber aumento no grau de satisfação, pois ao longo do projeto esses apresentaram maior desenvoltura, agilidade, domínio, equilíbrio, flexibilidade, resistência e força ao realizar movimentos. Outro aspecto evidenciado foi a importância de fazer exercícios em equipe que proporcionou aos participantes movimentarem-se em um ambiente de confiança, segurança e comodidade, possibilitado por meio de relações afetivas. Assim, pode-se concluir que as atividades físicas, na 
Atividades Interdisciplinares e Multiprofissionais: Relatos de Experiência com Participantes do Programa de Prevenção para Doenças Cardiovasculares

forma de vivências lúdicas, têm sido importantes recursos não medicamentosos na terapia e prevenção das doenças cardiovasculares, proporcionando aos participantes uma vida mais saudável (BONETTI, 2006).

A terapia holística aquática (watsu/ halliwick/biodanza aquática) foi conduzida com o objetivo de melhorar o condicionamento físico, diminuindo a tensão, ampliando movimentos e a respiração dos participantes. Uma avaliação realizada com vinte pacientes com doenças crônicas, com idades entre cinqüenta a setenta anos, no ano 2007, mostrou que o estado de estresse melhorou $70 \%$. Os aspectos mais representativos dessa melhora puderam ser observados nos depoimentos dos participantes e nas respostas ao questionário de estresse aplicado aos integrantes, os quais mostraram pela boa revigoração ao se levantar, pela queda de ansiedade e menor temor da perda do controle da mente, dos atos, palavras, pensamentos e sentimentos. Observou-se ainda melhora na postura e na disposição, na redução do estresse e da ansiedade, possibilitando um sono mais tranqüilo, além da melhora da consciência do corpo e o incentivo na busca da saúde espiritual (SCHUTZ et al.., 2009).

No atendimento psicológico, os participantes eram encorajados a relatar eventos e situações marcantes de sua vida sem constrangimentos ou censuras. $\mathrm{O}$ ambiente terapêutico acabou sendo um espaço em que a pessoa aprendeu a lidar melhor com suas emoções, seus sentimentos, suas crenças e seus conflitos pessoais e interpessoais. A descrição das contingências passadas ou vigentes criou condições para o grupo analisar o padrão atual de comportamento, ajudando os participantes a gerenciar, programar e produzir mudanças que ampliaram seu repertório comportamental (KOHLENBERG; TSAI, 2001; YALOM 2006; FRUTUOSO; CANTOS, 2008).

Verificou-se no atendimento psicológico em grupo que: 1) os conteúdos das queixas continham elementos comuns e isto ajudou os participantes a se identificarem uns com os outros; 2) as histórias de vida compartilhadas nos grupos serviram de modelo e fomentavam debates temáticos entre todos; 3) proporcionou aos membros encontrar soluções e indicá-las uns para os outros, promovendo um processo de ajuda mútua; 4) o surgimento de vínculo afetivo foi construído e mantido por interações de respeito num ambiente acolhedor de diferenças; 5) os participantes relatavam as mudanças comportamentais que ocorreram em suas vidas e as escolhas que fizeram 
Atividades Interdisciplinares e Multiprofissionais: Relatos de Experiência com Participantes do Programa de Prevenção para Doenças Cardiovasculares

durante o atendimento, o que servia de modelo para os demais se envolverem no controle de suas vidas com relação a aspectos alimentares, afetivos e profissionais (DELITTI; DERDAYK, 2008). Ao longo desses anos percebeu-se que a biodanza por meio da música, conto, expressão corporal nos encontros, repercutiu de forma integradora e afetiva nas interações dos membros que compõem o grupo. Os participantes relataram melhora dos sintomas e os enxames apontam melhora também no quadro clínico e laboratorial dos mesmos (CANTOS et al., 2008). Por exemplo, entre os anos 2002 a 2007 em torno de cento e cinquenta pessoas participaram do grupo de biodanza, durante pelo menos dois anos. Destas, vinte pessoas, compareceram com uma freqüência acima de $80 \%$ a todos os encontros, indicando um bom índice de adesão a longo prazo. Percebeu-se que esse grupo de biondaza comparado-o ao grupo controle (pessoas que participavam do projeto, mas não faziam biodanza), apresentou melhoras no perfil lipídico, bem como na glicemia de jejum e magnésio. Esses parâmetros bioquímicos tiveram boa correlação com estado de estresse (CANTOS, et al., 2005). Por outro lado, por meio das respostas do questionário de estresse e de depoimentos percebeu-se que as sessões de biodanza conduziram a melhoras no estilo e qualidade de vida dos seus integrantes, sobretudo na comunicação, na autoestima e também na aceitação do outro com todas as suas diferenças (CANTOS et al., 2008).

No desenvolvimento das vivências corporais lúdicas percebeu-se uma grande receptividade por parte dos participantes em função da diversidade de atividades que este tratamento proporcionou como danças, ginásticas, caminhadas, relaxamentos, jogos recreativos, capoeira, ioga, dentre outras. Observou-se, também, maior dinamismo nos movimentos e no relato verbal de bem-estar dos participantes. Além disso, foi possível notar a satisfação, pois ao longo do projeto eles apresentaram maior desenvoltura, agilidade, domínio, equilíbrio, flexibilidade, resistência e força ao realizar movimentos. Outro aspecto evidenciado foi a importância de fazer exercícios em equipe que proporcionou aos participantes movimentarem-se em um ambiente de confiança, segurança e comodidade, possibilitado por meio de relações afetivas (BONETTI, 2006).

A terapia holística aquática (watsu/ halliwick/biodanza aquática) os elementos mais representativos da melhora puderam ser observados no depoimento dos participantes e nas respostas ao questionário de estresse, os quais mostraram pela boa revigoração ao se levantarem, pela queda de ansiedade e menor temor da perda do controle da mente, dos atos, palavras, 
Atividades Interdisciplinares e Multiprofissionais: Relatos de Experiência com Participantes do Programa de Prevenção para Doenças Cardiovasculares

pensamentos e sentimentos. Uma avaliação realizada com 20 pacientes com doenças crônicas, idade entre 50 a 70 anos, no ano 2007 mostrou que o estado de estresse melhorou $70 \%$. Observou-se ainda melhoras na postura e na disposição, na redução do estresse e da ansiedade, possibilitando um sono mais tranqüilo, além da melhora da consciência do corpo e o incentivo na busca da saúde espiritual (SCHUTZ et al., 2009).

No atendimento psicológico os participantes eram encorajados a relatar eventos e situações marcantes de sua vida sem constrangimentos ou censuras. O ambiente terapêutico acabou sendo um espaço em que o indivíduo aprende a lidar melhor com suas emoções, seus sentimentos, suas crenças e seus conflitos pessoais e interpessoais. A descrição das contingências passadas ou vigentes criou condições para o grupo analisar o padrão atual de comportamento, ajudando os participantes a gerenciar, programar e produzir mudanças que ampliaram seu repertório comportamental (DELITTI; DERDAYK, 2008).

Verificou-se no atendimento psicológico em grupo que: 1) os conteúdos das queixas continham elementos comuns e isto ajudou os participantes a se identificarem uns com os outros; 2) as histórias de vida compartilhadas no grupo serviam de modelo e fomentava debates temáticos entre todos; 3) proporcionou aos membros encontrar soluções e indicá-las uns para os outros, promovendo um processo ajuda mutua; 4) o surgimento de vínculo afetivo foi construído e mantido por interações de respeito num ambiente acolhedor de diferenças; 5) os participantes relatavam as mudanças comportamentais que ocorreram em suas vidas e as escolhas que fizeram durante e atendimento o que servia de modelo par os demais se envolver no controle de sua vida relacionados com os aspectos alimentares, afetivos e profissionais (FRUTUOSO; CANTOS, 2009).

\section{CONSIDERAÇÕES FINAIS}

A adesão ao tratamento e conseqüentemente à eficácia da assistência prestada depende muito da percepção individual que cada um tem sobre seu estado de saúde e do comprometimento com as 
Atividades Interdisciplinares e Multiprofissionais: Relatos de Experiência com Participantes do Programa de Prevenção para Doenças Cardiovasculares

intervenções terapêuticas indicadas e adotadas. Sugere-se que amplie esta prática de associar medicina convencional com terapias alternativas no contexto da atenção à saúde, valorizando práticas que cuidem da pessoa como um organismo completo, nos aspectos físicos (corpo) e nos subjetivos e emocionais (psicológico).

É preciso lembrar que independentemente da terapêutica escolhida, a pessoa com doença crônica terá que constantemente lutar contra suas incapacidades e avançar no seu processo de cura, ou de redução de danos, para ter uma melhor qualidade de vida. Por outro lado, a condição de portador de uma doença crônica requer inserção em programas de tratamento ou reabilitação para facilitar a adaptação às novas condições e rotina de vida. Com isso, o paciente necessita de observação, controle e cuidados especiais da equipe de saúde (e de seus familiares) ao longo de toda sua vida. No que se refere à prevenção para doenças, esta experiência tem sido pioneira porque envolve a participação de uma equipe interdisciplinar e multiprofissional, com formações técnicas diversas, mas que trabalham juntos e conseguem, criar modelos de menores custos, que podem ser aplicados no Sistema Único de Saúde - SUS. Por outro lado, é necessário o investimento na educação continuada da equipe multiprofissional, para garantir a integralidade do cuidado em saúde e o reconhecimento e respeito às diferenças técnicas e teóricas existentes entre os membros da equipe multiprofissional.

As experiências aqui relatadas levam-nos a refletir sobre a importância de conscientizar a pessoa em relação ao seu estado de saúde, e a continuar com medidas de intervenção visando à prevenção das doenças cardiovasculares, a fim de se obter maior adesão dos participantes ao programa oferecido. Por isso, para trabalhar com esse tipo de população, é necessário pensarmos acerca do conceito de saúde e em como este conceito é percebido pelo paciente, sentido e entendido.

Destacamos a importância de envolver as pessoas com doenças crônicas em diferentes estratégias terapêuticas, cujo somatório implica na conscientização do indivíduo sobre seu estado de saúde, em seu engajamento nos processos de mudança, para sua adesão aos tratamentos disponibilizados, proporcionando uma vida mais saudável à população atendida. 
Atividades Interdisciplinares e Multiprofissionais: Relatos de Experiência com Participantes do Programa de Prevenção para Doenças Cardiovasculares

\section{AGRADECIMENTOS ${ }^{1}$}

\section{REFERÊNCIAS}

BONETTI, A. Vivências do coração. Dissertação de doutorado: Centro de Desportos. UFSC. Florianópolis. Dezembro, 2006.

BECKER, B. E.; COLE, A. J. Terapia aquática moderna. São Paulo: Manole, 2000.

CANTOS, G. A., et al. Avaliação do estado de estresse associado aos parâmetros bioquímicos de pacientes com dislipidemia, considerando o efeito terapêutico da biodanza. Rev. Pensamento Biocêntrico, v.1, p.1-5, 2008.

CANTOS, G.A et al. Biodanza como nova abordagem terapêutica para pacientes com problemas cardiovasculares. Rev. Pensamento Biocêntrico, v. 2, p.5-10, 2005.

CATANIA, A C. Aprendizagem: comportamento, linguagem e cognição. 4. ed. Porto Alegre: Artes Médicas, 1999.

CIPOLLE, R.J.; STRAND, L.M; MORLEY, P.C.. O exercício do cuidado farmacêutico. Tradução Denise Borges Bittar; Revisão técnica: Arnaldo Zubioli. Brasília: Conselho Regional de Farmácia, 2006. 396p.

CONEP - Comissão Nacional de Ética em Pesquisa. Normas para pesquisa envolvendo seres humanos: (Res CNS 196/96 e outras) = Rules on Research involving human subjects / Conselho Nacional de Saúde. Brasília: Ministério da Saúde, 2000.

DEL PRETTE, A.; DEL PRETTE, Z.A.P. Psicologia das relações interpessoais: vivências para o trabalho em grupo. Petrópolis, RJ: Vozes, 2009.

DELITTI, M.; DERDAYK. P. Terapia analítico-comportamental em grupo. Santo André, SP: ESETec Editores Associados, 2008

FRUTUOSO. J. T.; CANTOS G.A. Atendimento psicológico em grupos portadores de dislipidemia. In: Semana de psicologia da UFSC, 2009. p. 44-45, ago. de 2009. Anais... Florianópolis: UFSC, 2009.

Agradecimentos - A todos os integrantes, pacientes e profissionais do NIPEAD_HU_UFSC que contribuem ou contribuíram para o sucesso do tratamento oferecido, em especial à. Cláudia Souza Marquez da Silva, Carmen Waltrick (cardiologistas do Hospital Universitário da UFSC), Elizabeth M. Hermes (Bioquímica do HU-UFSC), .Jussara Gazzola (atual nutricionista da equipe, professora do Depto de Nutrição-UFSC), Maria da Graça Winckler Balen (Nutricionista HU-UFSC), Rodrigo Schütz e Claúdia Cavalett (Mestres em Farmácia-PGFAR), Jonathas Gomes de Medeiros e Renata Bittencourt (facilitadores de biodanza), Maria de Fátima da Silva Duarte (Dra. do Depto de Educação Física-UFSC). ${ }^{I}$ 
Atividades Interdisciplinares e Multiprofissionais: Relatos de Experiência com Participantes do Programa de Prevenção para Doenças Cardiovasculares

GOSTAWAMI, A. O médico quântico: orientações de um físico para a saúde e a cura. Ed. Cutrix, São Paulo, 2004.

KERBAUY, R.R. Comportamento e saúde. Sandro André, SP: ESETec Editores Associados, 2009.

KOHLENBERG, R. J; TSAI, M. Psicoterapia analítico funcional. Sandro André, SP: ESETec Editores Associados, 2001.

LEITE, I. C. Comparação das informações sobre as prevalências de doenças crônicas obtidas pelo suplemento saúde da PNAD/98 e as estimadas pelo estudo Carga de Doença no Brasil. Ciênc. Saúde Coletiva, v.7, n.4 , pp. 733-741, 2002.

LIPP, M. Pesquisa sobre estresse no Brasil: saúde, ocupações e grupo de risco. Campinas São Paulo: Papirus, 1996.

LIPP, M.; ROCHA, J. C. Stress, hipertensão arterial e qualidade de vida. Papirus Editora, São Paulo, 1996.

MALTA, D. C.; MERHY, E. E.. O percurso da linha do cuidado sob a perspectiva das doenças crônicas não transmissíveis. Interface , v. 14, n.34, pp. 593-606, 2010

MARTIN, G.; PEAT, J. Modificação de comportamento: o que é e como fazer. 8. ed. São Paulo:Roca, 2009.

MOLINA; M. C. B. et al. Hipertensão arterial e consumo de sal em população urbana. Rev. Saúde Pública, v.37, n.6, p. 743-750, 2003.

PASSOS, A. M., CANTOS, G.A. Análise qualitativa das preparações do cardápio de um grupo de prevenção para doenças cardiovasculares. In: Semana de Ensino, Pesquisa e Extensão, 7. Anais... Florianópolis: UFSC, 2009.

ROCHA M. E. et al. Associação das técnicas de watsu e halliwick com a biodanza aquática, como forma de melhorar o estresse psicológico de pacientes com doenças crônicas. Rev. Pensamento Biocêntrico, v. 9, p.69 -82, 2008.

ROSEIN, G. E., et al. Influência do estresse nos níveis sanguíneos de lipídios, ácido ascórbico, zinco e outros parâmetros bioquímicos. Acta Bioquím Clin Latino Am., v. 38,n. 1, p. 39-46, 2004.

SCHUTZ, R., et al. O método watsu e halliwick associados com a biodanza ${ }^{\circledR}$ : dados preliminares de uma população com doenças crônicas. Rev. Pensamento Biocêntrico. , v.10, p.3 - 15, 2009.

SERRANO, C. V. Jr, et al. Tratado de Cardiologia SOCESP. 2. ed. São Paulo: Manole, 2009. 
Atividades Interdisciplinares e Multiprofissionais: Relatos de Experiência com Participantes do Programa de Prevenção para Doenças Cardiovasculares

SILVA, L. M. C. da et al. Aposentados com diabetes tipo 2 na Saúde da Família em Ribeirão Preto, São Paulo - Brasil. Rev. Esc. Enferm. USP. v..44, n.2 , pp. 462-468, 2010.

TORO, R.A. Biodanza. Ed. Olavobrás, São Paulo-SP, 2002. 157 p.

SILVEIRA, M.S, et al. Fatores de risco para doenças cardiovasculares e suas associações com obesidade. Rev Ciências da Saúde, v.28, n.1, p 23-30, 2009.

SIMÃO, R. Fisiologia e prescrição de exercício para grupos especiais. São Paulo: Phorte, 2004.

VALLS L., T.I.; SILVA C. M M. El paciente como centro del seguimiento farmacoterapéutico. Esp, Pharmaceutical Care, v.10, n.3, p.120-130, 2008.

YALOM, I.D. Psicoterapia de grupo: teoria e prática. Porto Alegre, RS: Artmed, 2006.

\begin{abstract}
In this paper, we describe an experiment conducted by an interdisciplinary professional health team that focus on cardiovascular diseases' prevention The study included 480 people over a period 2007 to 2009 . They were patients with or without chronic diseases, most of them belonged to the university community. Initially, the patients had appointements with cardiologists and nutrionists and they could also attend lectures on topics related to the health-illness process. Later on, in order to combine conventional medicine and other health care promotion models, the team decided to offer a range of avaiable body practices to patients as alternatives in fighting the disease. These were biodanza, three different aquatic practices (watsu, halliwick and biodanza); body budic experience; ludic experiences of the heart. Furthermore, there was also a psychotherapy group and a nutritional education that involved lunching together and visiting the supermarket. The monitoring of these actions included primarily leveraged structured questionnaires, verbal reports, and clinical and biochemical analysis. Data was organized for further analysis in thematic categories involving aspects that had influenced the adherence of those people to the program. The participants reported as important that they became aware of how they were acting upon it. From this awareness, the participants could plan and be responsible for positive changes in their everyday attitudes that contributed to their better quality of life and psychological well-being. The combination of those different forms of treatment avaiable in the several therapeutical groups strengthened, for the patients, their humanist values associated with the physical and emotional recovery, integrating them better with the world, with other people and with themselves. This experience contributed to professional constructive knowledge and to the professional growth, valuing the contribution of the health team and it offered to its participants a model of care focused on health promotion and disease prevention.
\end{abstract}

Keywords: Cardiovascular Diseases, chronic diseases; Health promotion; Disease prevention; Alternative treatments; Health care models.

Originais recebidos em: 29/04/2010

Aceite para publicação em: 16/11/2010 